\title{
Physics of Plasmachemistry
}

\author{
Daniel C. Schram, Eindhoven
}

(University of Technology)

Traditionally, plasma physics has been stimulated by applications such as light production, interest in astrophysics and the prospect of thermonuclear fusion. In recent decades, the emphasis has been on hot hydrogenic plasmas in fusion devices, which has led to a deepening of our insight into transport phenomena in collisionless hot plasmas, and the development of new diagnostic techniques.

In the past few years, the physics of low temperature plasmas has received a new stimulus from applications ${ }^{1)}$ in chemistry and surface treatment that have emerged empirically as extrapolations of hot gas-chemistry and e.g. chemical vapour deposition (CVD). Impressive results have been obtained, but it is to be expected that further progress will require the characterization of the plasma, an understanding of the processes, along with advanced diagnostic techniques, and detailed descriptions of the plasma dynamics and excitation mechanisms. The non-equilibrium character of the plasma and its heating and confinement by electric and magnetic fields makes this essentially a physics area.

The plasma chemistry field can be sub-divided into three classes: first, volume chemistry in which a gaseous feed is converted to other products by means of a discharge, as e.g. ozone-production in corona discharges in the air; second, surface modification where the surface of a substrate is altered by bombardment with atomic particles ${ }^{2-4}$ ); third, melting and plasma spraying in which either material is melted by the heat of the plasma or surfaces are covered with particles of $10-100 \mu \mathrm{m}$ size accelerated and melted by the plasma ${ }^{5}$ ).

Here we shall concentrate on surface modification which is, at present, the most extensively explored field. To a large extent the work is motivated by developments in submicron technology and the demand for thin high quality layers with anticorrosive, hardness or other properties. The specific added value is high, which gives the incentive to invest, but in the long run more conventional applications may also become economically viable.

Examples of surface modification are: cleaning to remove adsorbed gases and impurities from surfaces (as discharge cleaning in tokamaks); improving the
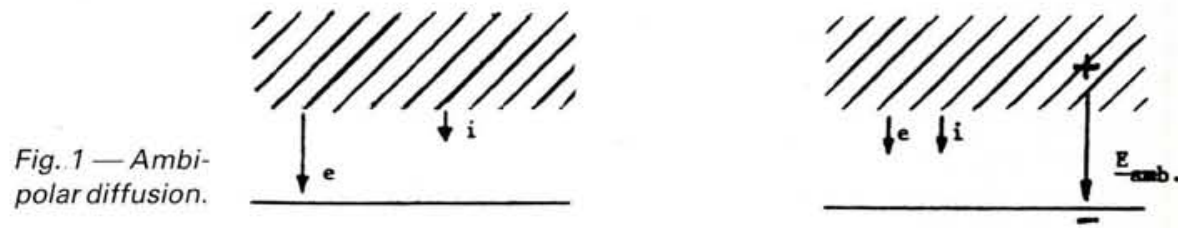

wettability of polymer surfaces; and etching, now widely used in the electronics industry, where advantage is taken of its anisotropy to produce large vertical as against horizontal etch rates. It is also possible to cover substrates with other materials and change the properties of the surface. The technique is important for the fabrication of solar cells, glassfibres, corrosion and wear-resistant layers and again for submicron technology. Freedom in the choice of materials, controllable substrate conditions and ambient pressure, associated with the non-equilibrium character of the plasma give special advantages to the technique. Other examples of surface modification are boriding, carbonizing and nitriding where atoms/ions are diffused into the substrate, to change the hardness of the surface without changing the mechanical tolerance.

Plasma polymerization is a related application; the polymers produced are highly cross-linked materials with high density and constitute new materials with novel properties.

From the examples quoted above it is evident that the primary reason for the use of plasmas is the production of a high local temperature which leads to a high specific reactivity, per unit volume as well as per particle (atom or molecule). The reactivity is usually determined by the electron temperature, $T_{e}$, which does not need to be in equilibrium with the heavy particle temperature. In most discharges, whereas the heavy particle or gas temperature is close to ambient, $T_{\mathrm{e}}$ is in the range of a few $\mathrm{eV}$ $\left(1 \mathrm{eV} \hat{=} 11.6 \times 10^{3} \mathrm{~K}\right)$. This feature enables one to have a high reactivity without the high bulk heat content of a system in chemical equilibrium. Moreover, the background gas may not only have a low temperature, but also a low density which has a definite side advantage for, e.g., deposition control. Also the temperature of the substrate to be treated or covered can be selected to maximise product quality e.g. the adhesion of deposited layers.

A definite advantage of plasmas is the large freedom of choice of material and form. The easiest is as a gas, but the material can also be introduced as a liquid in droplets or aerosols, or as a solid by sputtering or vaporization.

lonization of plasmas in the majority of cases is primarily induced by electron collisions, which lead to the production of all kinds of radical, whose densities

Fig. 2 - Plasma characterization; gd: glow discharge; ag: anomalous glow; a: arc; $t$ : thermal plasma.

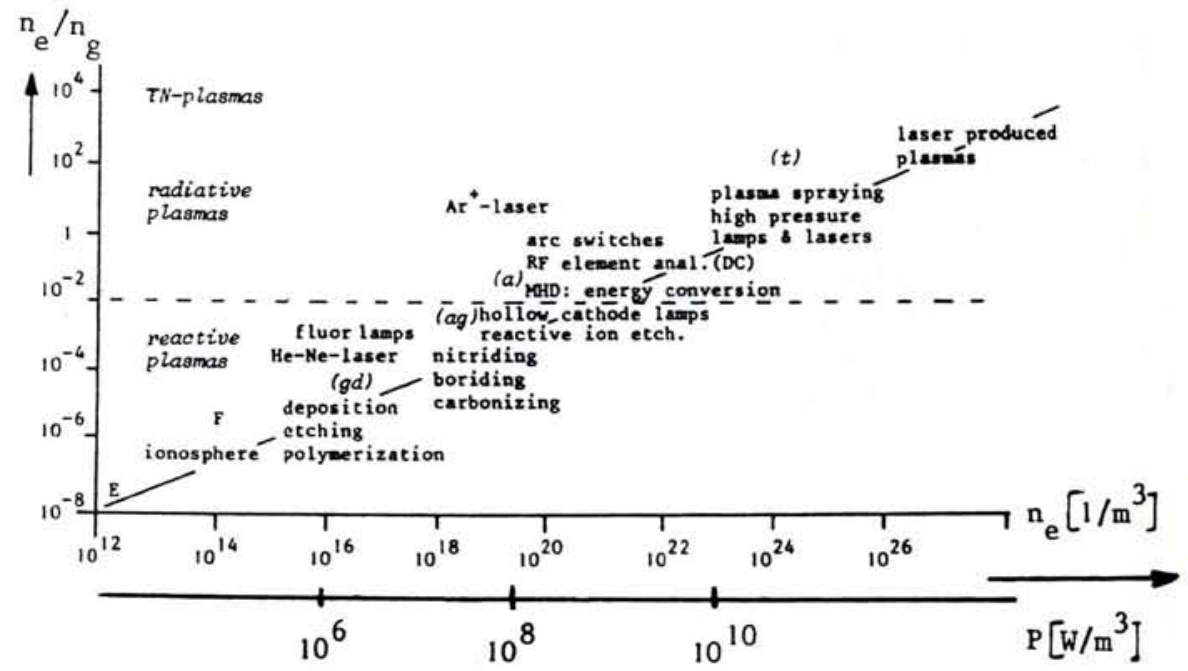


can be even higher than $n_{\mathrm{e}}$ and which have a high reactivity. Chemical equilibrium is by no means assured; only in high pressure, high power density plasmas may the system be close to local thermodynamic equilibrium.

Plasmas tend to maintain a quasi charge neutrality if negative ions are absent, i.e. on balance to have equal positive and negative charge densities:

$$
n_{\mathrm{e}}=n_{\mathrm{i}}
$$

Only in a layer adjacent to the wall may a substantial electric field build up caused by a small excess of positive charges in the discharge (Fig. 1). Electrons tend to diffuse out of the discharge faster than the colder and much heavier ions, creating a space charge that opposes the effect until equilibrium is established with ions and electrons diffusing together so-called ambipolar diffusion. The thickness of the sheath adjacent to the wall where the imbalance in electron and ion densities occurs is related to the Debye shielding length, defined as:

$\lambda_{\mathrm{D}}=\left(\varepsilon_{\mathrm{o}} k T_{\mathrm{e}}\right)^{1 / 2} / n_{\mathrm{e}} e^{2}$

$\cong 7 \times 10^{3}\left(T_{\mathrm{e}}[\mathrm{eV}] / n_{\mathrm{e}}\right)^{1 / 2}\left(10^{4} / n_{\mathrm{e}}{ }^{1 / 2}\right)$

Typical values are $100 \mu \mathrm{m}$ for low density plasmas and $0.1 \mu \mathrm{m}$ for very high density plasmas. This sheath and its associated potential difference which may accelerate ions to the wall is very important in plasma processing and is an essential factor in the unidirectionality of plasma etching.

If the plasma is heated by RF electric fields, the RF only partly penetrates the plasma, but still enough to heat the electrons. Most of the field is concentrated over the sheath.

The character of the plasma used varies enormously, but up to now, no systematic guide to selection has been produced. Trial and error has been the practice with no certainty that the choice is optimum. This becomes clear from Fig. 2 in which several plasmas are displayed in a $n_{\mathrm{e}} / n_{\mathrm{g}}, n_{\mathrm{e}}$ diagram. Here $n_{\mathrm{e}} / n_{\mathrm{g}}$ is the ratio of the electron to the neutral gas density, a parameter that is more appropriate than the electron temperature, since the latter is always between $1 / 4-1 / 15$ of the ionization potential (from low to high pressure discharges).

It is observed that all non-magnetically confined plasmas group roughly along the line:

$n_{\mathrm{e}} / n_{\mathrm{g}} \cong\left(10^{-24} n_{\mathrm{e}}\right)^{1 / 2}$ where $n_{\mathrm{e}}$ is in $\mathrm{m}^{-3}$ (3) This is consistent with the principle that the Debye length, $\lambda_{D}$, has to be substantially smaller (100 times) than the ionneutral mean free path, $\lambda_{\text {io }}$. Apparently, the ambipolar electric field must be sufficiently small for the plasma to exist, at least in the ionizing phase.

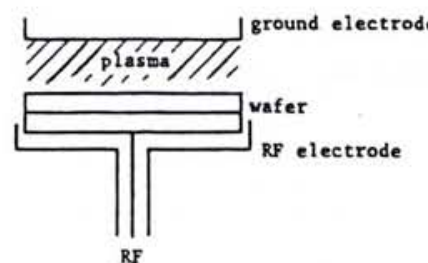

(a)

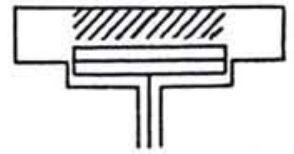

(b)

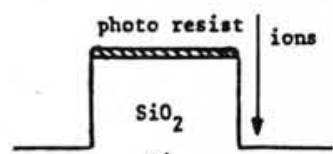

Si

(c)

Fig. 3-Schematic view of a single wafer etch apparatus: (a) normal set-up, (b) enclosed in microwave cavity, (c) detail of etch structure.

In magnetically confined plasmas, the constraints are different and a higher degree of ionization is achievable at lower electron densities. We shall return to the constraints imposed on plasmas in the next section.

\section{Plasma Treatment in situ: \\ Plasma Etching}

In plasma etching and plasma deposition, normally RF plasmas are used with pressures around $10 \mathrm{~Pa}$, power densities of $1 \mathrm{~W} / \mathrm{cm}^{3}$ and frequencies from the audio range to tenths of megahertz. A schematic view of a prototype single wafer etcher is given in Fig. 3 . It consists of two parallel plates, $6 \mathrm{~cm}$ in diameter the distance between which is varied from $1-3 \mathrm{~cm}^{7}$ ). The plasma is created by ionization, radicals are formed by dissociation and vibrational excitation takes place, mostly by electrons.

From the plasma, diffusive flows impinge on the surface, the ions being accelerated often by the self bias voltage of ambipolar origin. To study the physics at the surface, one needs to know the net flows to the surface: for the ions this is the incident flow; for the radicals it is the difference between incident and desorption flows, the latter depending on the "sticking efficiency" at the surface. What happens to the vibrational excitation of molecules is still a matter of guess-work. It is quite possible that highly vibrationally excited molecules dissociate at the surface and contribute to the formation of radicals adsorbed at the surface.

The chemistry at the surface is dependent on the composition of adsorbed species and the influx of ions of typically $100 \mathrm{eV}$ energy, which determine the anisotropy of the etching. Schematically the situation can be summarized as indicated in Fig. 4. As a first working hypothesis, an adsorbed gas layer is postulated. The reactivity at the surface can then be roughly estimated from the composition of fluxes to the surface, the sticking probability and an effective temperature, $T_{\text {eff }}$, of the gas layer, which defines the chemical composition and the desorption of the radicals. The plasma physics of the problem can then be reduced to calculating the net fluxes to the surface. For simplicity we ignore the role of photons.

In the plasma production volume (I) the reacting fluxes are produced which cross the drift volume (II) (in this example the same volume as I) and lead to alterations of the substrate surface (IV) through chemical reactions in the gas adsorbed layer (III).

In the etching process, three requirements have to be met simultaneously:

a) a fast etching rate,

b) high selectivity, i.e. the material to be processed should be etched much faster than other materials (e.g. protective resists),

c) good anisotropy especially if structures on a scale in the order of $\mu \mathrm{m}$ are to be achieved.

Usually, there is a trade-off, and it is clear that the composition of radical flow and the energy of the ions impinging on the substrate are crucial.

With ion mass spectrometry, the ions can be identified and their energy can be estimated from retarding field analysis. With an additional microwave cavity the electron density can be determined from the detuning of cavity modes. With actinometry, i.e. from intensity ratios of e.g.

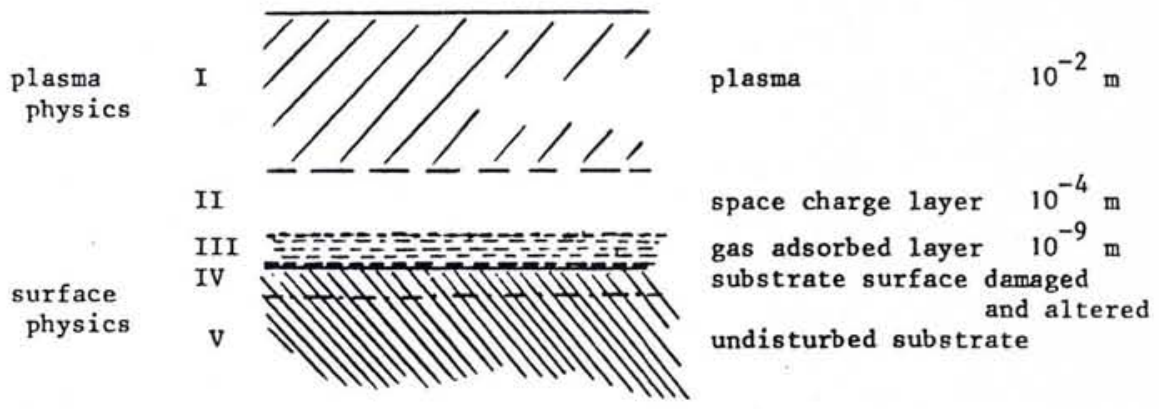

Fig. 4 - Schematicized sub-division of plasma surface modification. 


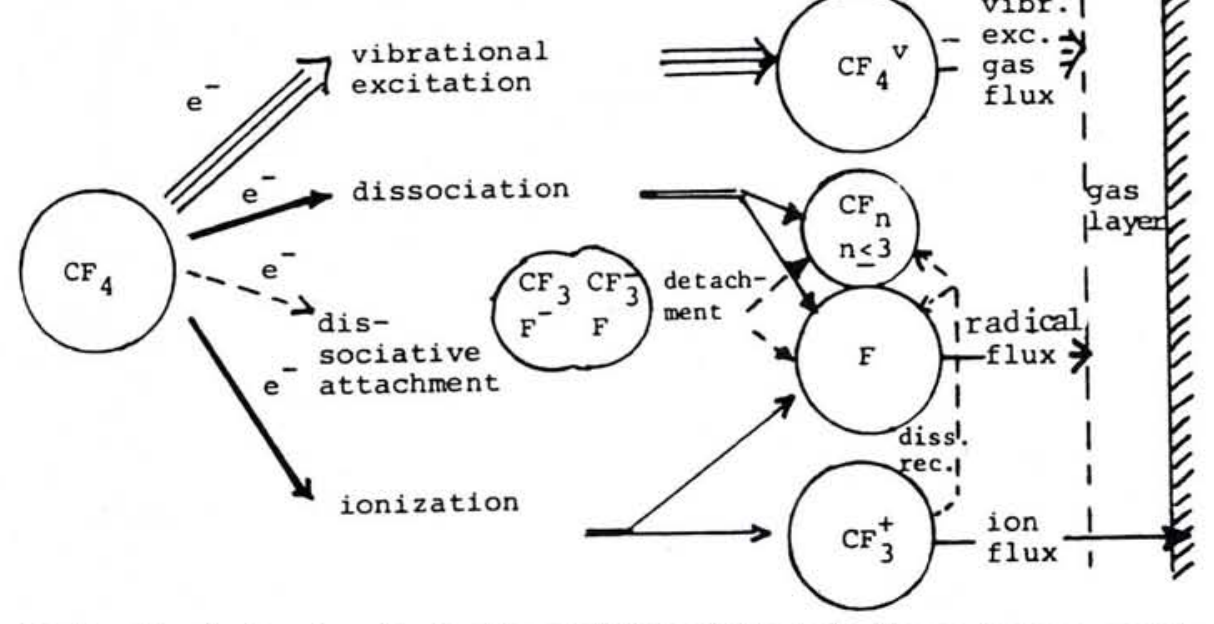

Fig. 5 - The dominant reaction kinetic path (full lines). The dashed lines indicate sequential reactions which may be important.

F and Ar spectral lines, species density can be estimated and with ellipsometry the etching rates can be measured in situ. So, the etching process can be subdivided into the following processes:

1. Ionization and creation of electrons; the energy distribution is of importance. 2. Production of radicals.

3. Fluxes of ions and radicals to the substrate and adsorbed gas layer.

4. The composition of the adsorbed gas layer.

On the kinetics at the surface, different opinions exist. In the etching of $\mathrm{SiO}_{2}$ by $\mathrm{CF}_{4}$, one possibility is the direct etching by fluor atoms, whilst the carbon atoms take care of the $\mathrm{O}$-atoms in the $\mathrm{SiO}_{2}$-substrate. In another view, the dominant reaction is $2 \mathrm{CF}_{2}+\mathrm{SiO}_{2} \rightarrow$ $\mathrm{SiF}_{4}+2 \mathrm{CO}$. The latter is any way the representation of the overall balance.

In this picture the selectivity with respect to pure silicon is obtained through inhibition by polymer formation. There are two hypotheses on the mechanism of anisotropy. One is basically of a chemical nature: only by ion bombardment is the inhibition of etching by polymer formation broken down. The other explanation is of a physical nature: in that picture the ions damage the surface thereby facilitating the etching reaction.

Instead of elaborating on all the conversion processes in the plasma we shall follow here the line of dominant kinetics (Fig. 5). We assume that the density of negative ions is much smaller than the electron density $n_{\mathrm{e}}$ (as can also be concluded from a full numerical analysis) so that $n_{\mathrm{e}}$ is equal to the ion density and the attachment-detachment channel can then be considered as a dissociation.

In the main, $\mathrm{CF}_{3}{ }^{+}$is the dominant ion, since its production is more effective and the ambipolar dwell time is too short for the probability of loss processes like dissociation, dissociative recombination etc. to be significant. However, side reactions are of importance for the production of other radicals like $\mathrm{CF}, \mathrm{CF}_{2}$, $\mathrm{CF}_{3}$. In our (still unproven) hypothesis, the actual chemical composition of the plasma is irrelevant, so long as sufficient reactants are transmitted to the adsorbed layer and the effective temperature and reactivity there are adequate for the etching process to take place. We only need to provide enough reactants, enough $\mathrm{C}$ and $\mathrm{F}$ particles in whatever form, and ions with sufficient energy to promote the process.

In this respect we note that any dissociation activity (ionization of $\mathrm{CF}_{4}$ to $\mathrm{CF}_{3}{ }^{+}$, dissociative recombination, dissociation of $\mathrm{CF}_{4}$ ) produces at least one $\mathrm{F}$-atom. For the production of $\mathrm{CF}_{2}$, only dissociative recombination of $\mathrm{CF}_{3}{ }^{2}$ and dissociation of $\mathrm{CF}_{n}, n \geq 3$ are possible channels. Moreover $\mathrm{CF}_{2}$ can be dissociated again, whereas $F$ cannot. So in a first rough estimate we shall assume that we need only to follow the kinetics of $\mathrm{F}$ and $\mathrm{CF}_{3}{ }^{+}$.

The $\mathrm{CF}_{3}{ }^{+}$ion ambipolar flux can be obtained from the mass balance:

$$
n_{\mathrm{e}} n_{\mathrm{CF} 4} k_{\text {ion }}=D_{\mathrm{A}} n_{\mathrm{e}} / \mathrm{L}^{2}
$$

where the volume production is equated to the divergence of the ambipolar flow, $D_{A}$ is the ambipolar diffusion coefficient and $L$ is the scale length of the electron density. The rate coefficient for ionization, $k_{\text {ion, }}$ depends sensitively on the electron energy distribution $f\left(V_{\mathrm{e}}\right)$. Several treatments show distribution functions which differ from the Maxwellian, but for which the underpopulation of the tail is less severe than in DC discharges. Apparently stochastic RF heating of electrons takes place especially for large values of the ratio of RF electric field to the plasma pressure.

Effective ionization electron temperatures of $3-6 \mathrm{eV}$ are found in numerical analysis ${ }^{8}$ ). The product $D_{\mathrm{A}} n_{\mathrm{CF} 4}$ depends only (though more weakly) on the electron distribution function. So, from Eq. (4) by equating $n_{\mathrm{e}}$ and $n_{\mathrm{CF} 4}$ we can conclude immediately that the effective electron temperature depends only on the product $n_{\mathrm{CF}_{4}} L$ as:

$n^{2}{ }_{\mathrm{CF} 4} L^{2}=\left(D_{\mathrm{A}} n_{\mathrm{CF} 4}\right) / k_{\text {ion }}$

$$
\text { = function }\left(f\left(V_{e}\right)\right)
$$

Apparently, there is an interrelation between $T_{\mathrm{e}}^{\text {eff }}$ and the pressure and scale length, and from the mass balance only a limited range of $n_{\mathrm{CF} 4} L$ is permitted. The ion flux can now be obtained by integrating the mass balance over the plasma volume and using Gauss' theorem. With realistic values of the plasma parameters $\left(n_{\mathrm{e}} \cong 10^{16} / \mathrm{m}^{3}, T_{\mathrm{e}}^{\text {eff }} \cong 4 \mathrm{eV}\right.$, $\mathrm{p} \cong 20 \mathrm{~Pa}, L \cong 1 \mathrm{~cm}$ ) we obtain fluxes of $0.5 \times 10^{20} / \mathrm{m}^{2} \mathrm{~s}$.

The radical flux can be estimated in the same way. Since with any ionization, one F-atom is produced and also dissociation leads to F-production, the net radical flux will be somewhat larger than the ion flux. So, the ion flux is probably the rate limiter which is supported by the observation that the etch rate varies linearly with electron density (Fig. 6).

The energy balance imposes another constraint on the plasma. The energy input must balance the losses by ionization, dissociation and vibrational excitation. As dissociation is only slightly more probable than ionization and much more energy is involved in the latter, dissociation losses can be ignored in an overall energy balance. This is not so for vibrational losses; usually they are comparable with the energy loss involved with the production of ions and acceleration in the sheath-voltage drop.

The energy balance indicates that the electron density is roughly proportional to the power density. With the usual value of $1 \mathrm{~W} / \mathrm{cm}^{3}$, values of $10^{16} / \mathrm{m}^{3} \mathrm{can}$ be expected and are observed with

Fig. 6 - Etch rate and electron density as a function of RF power.

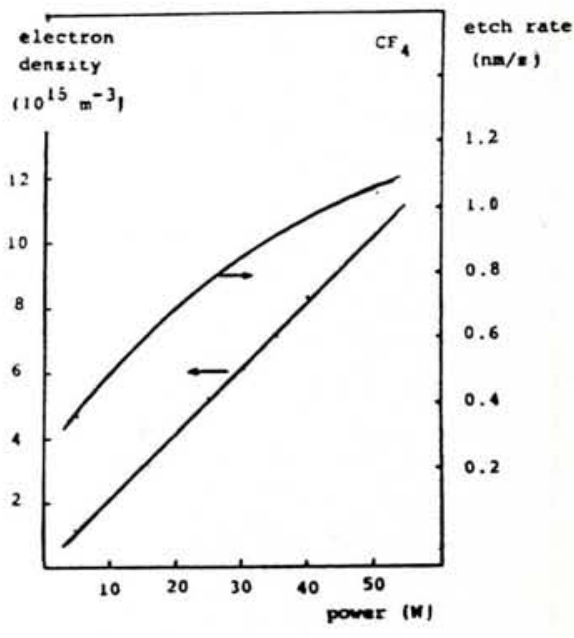


microwave detuning techniques $\left.{ }^{6}\right)$. The main energy flow to the wall must be carried by ions accelerated in the sheath and by vibrationally excited $\mathrm{CF}_{4}$ molecules. If we assume vibration energies of a few tenths $\mathrm{eV}$ and cross-sections of $10^{-19} \mathrm{~m}^{2}$, the energy flow can be explained, if we also accept that a substantial fraction of the $\mathrm{CF}_{4}$ molecules are vibrationally excited. If we assume that $\mathrm{CF}_{4}^{\mathrm{V}}$ contributes to the gas layer, then the supply of chemical species is to a large extent taken care of by vibrationally excited $\mathrm{CF}_{4}$ molecules. Schematically, the situation can then be described thus:

- ion flow is rate limiting and reaches the etch surface;

- radical flow and $\mathrm{CF}_{4}^{\mathrm{V}}$ flow contribute to the $\mathrm{C}$ and $\mathrm{F}$ content in the gas adsorbed layer;

- the energy flow carried by ions and $\mathrm{CF}_{4}^{\mathrm{V}}$ determine the effective temperature of the gas layer (over and above external heating of the substrate of course) and therefore the composition of radicals in the layer. The situation can be influenced by increasing the energy flow, or heating the substrate to promote etching, flushing the electrode with unexcited $\mathrm{CF}_{4}$, and cooling to promote deposition and/or polymerization.

We conclude, that by postulating an adsorbed gas layer, the physics of the gas discharge can be largely schematicized; attention should now be focussed on the physics of the adsorbed gas layer at the surface, in the presence of a gas discharge with the associated polarization fields, ion fluxes, vibrational excitation and radical flows. Even though the present picture is only approximate, it clearly demonstrates the constraints in the active plasma. Pressure, scale lengths, electron energies are interrelated and the power density determines the electron density and therefore the achievable ion fluxes, and etch rates. With plasma dimensions of a few $\mathrm{cm}$ the working pressure is restricted to the 10-100 Pa range.

These limitations are less severe, or even absent if the plasma production is separated geometrically from the plasma treatment volume. In that case the rate is determined by the number of active particles, usually ions, arriving at the surface. As recombination of the ions contributes to the ambient pressure this places limits on flow and etch rates, but even so higher levels can be attained.

In the above, the physics of plasmachemistry has been illustrated through the intermediary of an etching process. Many other ways of producing plasmas can be used, such as inductive coupling, magnetron discharges, etc. but this

\section{Université Louis Pasteur Strasbourg — France}

\section{Microélectronique Optoélectronique}

L'UNIVERSITÉ LOUIS PASTEUR de Strasbourg souhaite développer des domaines de recherche en microélectronique, optoélectronique et systèmes. Plusieurs postes de professeurs et de maîtres de conférences sont à pourvoir. Les secteurs de recherche en cours de développement sont:

- pour la microélectronique: recherche et développement de capteurs intégrés et circuits associés, architecture interne de ces dispositifs (C.A.O.), destinés, en particulier, à la physique des particules.

- pour l'optoélectronique: interfaçage de systèmes optiques bistables et insertion dans les systèmes numériques.

— pour les systèmes: architecture des systèmes pour l'automatique et le traitement d'images.

Les laboratoires engagés dans ces activités sont:

$\star$ Laboratoire de Physique et Applications des Semiconducteurs

$\star$ Laboratoire de Spectroscopie et Optique du Corps Solide

* Laboratoire des Sciences de l'Image et Télédétection.

$L^{\prime}$ enseignement sera donné dans le cadre des $2^{e}$ et $3^{e}$ cycles de Physique, ainsi qu'à I'Ecole d'Ingénieurs Physiciens et à I'I.U.T. Louis Pasteur.

Pour tous renseignements, écrire à:

Madame COULIBALY, Institut de Physique

3, rue de I'Université, F-67000 STRASBOURG (FRANCE) example illustrates clearly the large variety of processes and subsequent physical and chemical problems to be solved.

\section{REFERENCES}

1. A. Rutscher and H. Deutsch (Eds.), Wissenspeicher Plasmatechnik, (Fachbuchverlag, Leipzig) 1983.

2. Bell A.T., Plasma Chemistry III, Eds. S. Veprek and M. Venugopalan, Topics in Current Chemistry 94 (Springer Verlag) 1980. 3. Kay E, Coburn J. and Dilks A., Plasma Chemistry III, Eds. S. Veprek and M. Venu- gopalan, Topics in Current Chemistry 94 (Springer Verlag) 1980.

4. D'Agostino R. et al., Pure and Appl. Chem. 57 (1985) 1287 (ISPC-7).

5. Fauchais P. et al., Pure and Appl. Chem. 57 (1985) 1171 (ISPC-7).

6. Bisschops T.J. and de Hoog F.J., Pure and Appl. Chem. 57 (1985) 1311 (ISPC-7).

7. Edelson D. and Flamm D.L., J. Appl. Phys. 56 (1984) 1522.

8. Vallinga P.M. et al., Proc. Symp. on Plasma Chemistry (ISPC-7), Eindhoven (1985) 1399.

9. Zalm P.C., Pure and Appl. Chem. 57 (1985) 1253 (ISPC-7),

\section{Professor J.J. Went Leaves Us}

Readers will be sad to learn of the recent death of Professor J.J. Went of Arnhem just before his 79th birthday. Secretary of the EPS and member of the Editorial Board of Europhys. News from 1973-75, member of the Executive Committee until 1977, he was a major influence in restructuring the Society during its most difficult period. A nuclear physicist with a strong belief in nuclear power, his courteous determination was a major factor in ensuring the Society's survival. 